\title{
Regulation of DNA damage response and cell cycle in radiation-resistant HL60 myeloid leukemia cells
}

\author{
MASAHARU HAZAWA, YOICHIRO HOSOKAWA, SATORU MONZEN, \\ HIRONORI YOSHINO and IKUO KASHIWAKURA \\ Department of Radiological Life Sciences, Division of Medical Life Sciences, \\ Hirosaki University, 66-1 Honcho, Hirosaki, Aomori 036-8564, Japan
}

Received January 23, 2012; Accepted March 16, 2012

DOI: $10.3892 /$ or.2012.1771

\begin{abstract}
The acquisition of resistance to radiation has been a matter of concern in clinical cases. However, mechanisms underlying the acquisition of radiation resistance are yet to be elucidated. We established a radiation-resistant strain (ResHL60 cells) from HL60 leukemic cells and investigated their response to radiation. Res-HL60 cells not only proliferated on the fifth day after radiation but also had a high survival rate in a clonogenic assay. Although Chk1 was activated in HL60 cells after irradiation, the expression levels of Chk1 in Res-HL60 cells decreased. There were few differences between the two cell lines with regard to Chk2 activity. Res-HL60 cells show prolonged $\mathrm{G} 2 / \mathrm{M}$ arrest and an early decrease in the extent of DNA damage. However, inhibitors against ATM/ATR and DNA-dependent protein kinase (DNA-PK) both abrogated the radiation resistance capacity of the cells. These results reveal that radiation-resistant strains have a high repair capacity, and inhibition of the repair system at an early stage is an effective strategy in the second round of radiation therapy.
\end{abstract}

\section{Introduction}

The acquisition of resistance to radiation is a matter of concern in clinical cases; even so, radiation therapy is an effective procedure for the treatment of cancer. Tumor cells are heterogeneous with respect to radioresponsiveness. The radioresistant phenotype is ascribed to several factors, including alterations in cell cycle checkpoints, delayed growth, and a reduced frequency of apoptosis (1). Unfortunately, the mechanisms underlying the acquisition of resistance to radiation need to be elucidated.

Correspondence to: Professor Yoichiro Hosokawa, Department of Radiological Life Sciences, Division of Medical Life Sciences, Hirosaki University, 66-1 Honcho, Hirosaki, Aomori 036-8564, Japan

E-mail: hosokawa@cc.hirosaki-u.ac.jp

Key words: radiation, radiation resistance, cell cycle, DNA damage, HL60
Sensitivity to radiation differs among cells and depends on the cell type and tissue type. According to the BergonieTribondeau law, highly proliferative and immature populations are sensitive to ionizing radiation. It is well known that radiation targets DNA, and double-strand breaks (DSBs) are critical to DNA damage in eukaryotic cells (2). For overcoming damage to DSBs, there exist DNA repair mechanisms such as homologous recombination (HR) repair and non-homologous end-joining (NHEJ) repair (3).

Because each repair process depends on the existence of sister chromatin, the HR activity increases during the S and G2 phases, and NHEJ is predominant in the G0 and G1 phases (4). Ataxia telangiectasia mutated (ATM) is a member of the phosphoinotidylinositol-3-kinase (PIK) family and has a crucial role in DNA damage responses, activation of DNA repair, and checkpoint- and cell cycle-regulating proteins (5). Especially, ATM promotes repair of DSBs by HR. In contrast, DNA-dependent protein kinase (DNA-PK) is another phosphoinositol kinase (PIK) family member recruited by Ku to DSBs. DNA-protein kinase catalytic subunit (PKcs) promotes NHEJ and phosphorylates itself and other repair proteins (6). Proteins mediating HR and NHEJ are largely distinct, but some have been shown to participate in both pathways.

In a previous study, it was demonstrated that only 26 out of 110 tumor cell lines showed a positive correlation between radiation sensitivity and radiation-induced DSBs (7). However, another study showed that 89 out of 101 tumor cell lines had a positive correlation between radiation sensitivity and the yield of unrepaired DSBs (8). Therefore, the repair of DSBs is likely to determine radiation sensitivity. However, there are few reports that have investigated the radiation sensitivity and acquisition of resistance to radiation in cells derived from their original cell line (9).

In the present study, we focused on the acquisition of radiation resistance in HL60 myeloid leukemia cells. As an imitation of the model of radiation-resistant tumor cells subjected to radiation, we exposed HL60 cells to radiation at 16 Gy (4 Gy per week $\mathrm{x} 4$ ), and cloned the cells that survived. Cells thus established were named resistant-HL60 (Res-HL60) cells. To understand the mechanism underlying acquisition of resistance to radiation, we investigated the properties of HL60 and Res-HL60 cells and the potential contributors of radiation resistance. 


\section{Materials and methods}

Reagents. Caffeine, an inhibitor of ataxia telangiectasia mutated (ATM) kinase, was purchased from Sigma (St. Louis, MO) and was diluted in water to a concentration of $200 \mathrm{mM}$. NU7026, a specific inhibitor of DNA-PK, was purchased from Sigma and was diluted in dimethyl sulfoxide (DMSO) (Wako, Osaka, Japan) to a concentration of $5 \mathrm{mM}$. Caffeine and NU7026 were always used at concentrations of $2 \mathrm{mM}$ and $5 \mu \mathrm{M}$, respectively.

Antibodies. Phospho-Chk1 (Ser 296) Ab (rabbit no. 2349), phospho-Ch2 (Thr 68) Ab (rabbit no. 2661), and phospho-ATM (Ser 1981) (D6H9) mAb (rabbit no. 2883) were purchased from Cell Signaling Technology (Tokyo, Japan). DNA-PKcs (Ser 2056) Ab (Rabbit ab18192) was purchased from Abcam (Tokyo, Japan).

Cell line. The human tumor cell line HL60 (promyelocytic leukemia cells) was purchased from the RIKEN BioResource Center (Tsukuba, Japan). Radiation-resistant HL60 (Res-HL60) cell line was established by subjecting to 4 Gy irradiation/ week for 4 weeks. HL60 and Res-HL60 cells were maintained in RPMI-1640 medium (Gibco ${ }^{\circledR}$ Invitrogen, Carlsbad, CA) supplemented with $10 \%$ heat-inactivated fetal bovine serum (Bio Serum, Hiroshima, Japan) and 1\% penicillin/streptomycin (Gibco Invitrogen) in a humidified atmosphere at $37^{\circ} \mathrm{C}$ and $5 \%$ $\mathrm{CO}_{2}$.

In vitro irradiation and surviving fraction. HL60 and Res-HL60 cells were irradiated with X-rays $(150 \mathrm{kV}, 20 \mathrm{~mA})$ using $0.5-\mathrm{mm}$ aluminum and $0.3-\mathrm{mm}$ copper filters at a distance of $45 \mathrm{~cm}$ from the focus at a dose of 95-100 cGy/min (MBR-1520R; Hitachi Medical Corp., Tokyo, Japan) in the range of 1-8 Gy. During $\mathrm{X}$-ray exposure, the dose intensity was evaluated using the probe with ionization chamber. Irradiated cells were cultured using the plasma clot technique described in a previous report (10) at $37^{\circ} \mathrm{C}$ in a humidified atmosphere containing $5 \% \mathrm{CO}_{2}$. On the eighth day from the start of irradiation, colonies consisting of $>50$ cells were counted using an inversion microscope.

Growth inhibition assay. Cells were seeded in a $35-\mathrm{mm}$ culture dish (Iwaki) with $2 \mathrm{ml}$ of culture medium at $1-1.5 \times 10^{5}$ cells $/ \mathrm{ml}$. After determined periods of culture, cells in each condition were harvested, and diluted in trypan blue (Nacalai Tesque, Kyoto, Japan) to help distinguish between viable cells and damaged or dead ones. Cell growth was investigated by microscopic observations, and counting of viable cells by means of a Burker-Turk hemocytometer (SLGC, Saitama, Japan).

Cell cycle analysis. HL60 and Res-HL60 cells were seeded in a $35-\mathrm{mm}$ culture dish with $2 \mathrm{ml}$ of culture medium at $1 \times 10^{5}$ cells/ $\mathrm{ml}$ with or without either one of the specific inhibitors and were pre-incubated for $2 \mathrm{~h}$. After the cells were irradiated at $4 \mathrm{~Gy}$, they were incubated for $24 \mathrm{~h}$. The harvested cells were treated with phosphate-buffered saline (PBS) containing $0.1 \%$ Triton X-100 (Wako) for $5 \mathrm{~min}$ on ice, and stained with propidium iodide (PI; $50 \mu \mathrm{g} / \mathrm{ml}$ ) for $30 \mathrm{~min}$ in the dark. Analysis of the cell cycle distribution was performed using a Cell Lab Quanta ${ }^{\mathrm{TM}}$ Sc MPL (Beckman-Coulter, CA).
Detection of active caspase-3. The detection of active caspase-3 was performed using fluorescein isothiocyanate (FITC)conjugated monoclonal active caspase-3 antibody apoptosis kit I (BD Bioscience, San Jose, CA, USA) according to the manufacturer's instructions. Briefly, each cell was washed twice with ice-cold PBS and suspended in Cytofix/Cytoperm ${ }^{\mathrm{TM}}$ solution. After incubation on ice for $20 \mathrm{~min}$, the cells were pelleted, aspirated, and then rinsed with wash buffer at room temperature. Each cell was suspended in perm/wash buffer containing 5\% (v/v) FITC-conjugated antiactive caspase-3 Ab for $40 \mathrm{~min}$ at room temperature in the dark. The cells were rinsed with perm/wash buffer and analyzed using Cell Lab Quanta SC MPL.

$\gamma$-H2AX measurement by flow cytometry. HL60 and Res-HL60 cells were irradiated (4 Gy). At 10, 20, 30, 40, 60, 90, and 120 min after irradiation with X-rays, the cells were fixed in icecold $75 \%$ ethanol and stored at $-20^{\circ} \mathrm{C}$ for $>6 \mathrm{~h}$. The fixed cells were washed with PBS containing $1 \%$ bovine serum albumin (Sigma) (PBS-BSA) and treated with PBS-BSA containing $0.2 \%$ Triton-X 100 on ice for $5 \mathrm{~min}$. After washing, the cell pellets were incubated with anti- $\gamma-\mathrm{H} 2 \mathrm{AX} \mathrm{Ab}$ (mouse, monoclonal IgG1) (Upstate, Temecula, CA) in PBS-BSA containing $0.2 \%$ Triton- $X$ at room temperature for $1 \mathrm{~h}$. After washing, the cells were treated with a donkey anti-mouse IgG-FITC (Santa Cruz) in PBS-BSA containing 0.2\% Triton X-100 for $30 \mathrm{~min}$ in the dark. Thereafter, the stained cells were analyzed using Cell Lab Quanta Sc MPL.

SDS-PAGE and western blot analysis. SDS-PAGE and Western blotting was performed as described in a previous report (11). Briefly, cells were treated with each compound in the medium and incubated for $24 \mathrm{~h}$. Harvested cells were lysed with $50 \mathrm{mM}$ HEPES- $\mathrm{HCl}$ (pH 7.4), $100 \mathrm{mM} \mathrm{NaCl}, 1 \%$ Triton X-100, and $1 \mathrm{mM}$ phenylmethylsulfonyl fluoride (PMSF; Wako) on ice for $30 \mathrm{~min}$ and subjected to sonication twice for $30 \mathrm{sec}$ at ice-cold temperature. After centrifugation at $12,000 \mathrm{rpm}$ for $20 \mathrm{~min}$ at $4^{\circ} \mathrm{C}$, the protein concentration in the supernatant was determined by using the Bio-Rad protein assay kit (Bio-Rad Lab, Hercules, CA, USA) using the SmartSpec ${ }^{\mathrm{TM}}$ Plus spectrophotometer (Bio$\mathrm{Rad})$. An equal volume of sample buffer $(625 \mathrm{mM}$ Tris- $\mathrm{HCl}$, $\mathrm{pH} 6.8,20 \%$ SDS, $2 \%$ 2-mercaptoethanol, $2 \%$ glycerol) was added to the supernatant, which was then boiled for $5 \mathrm{~min}$. Proteins $(40-80 \mu \mathrm{g})$ were separated by SDS-PAGE and transferred onto nitrocellulose membranes (Advantec Toyo, Tokyo, Japan). The membrane was reacted with each primary antibody in Tris-buffered saline (10 mM Tris- $\mathrm{HCl}, \mathrm{pH} 7.4,100 \mathrm{mM} \mathrm{NaCl}$, $0.1 \%$ Tween-20) supplemented with $5 \%$ non-fatty milk or bovine serum albumin for $1 \mathrm{~h}$ at room temperature after blocking of the membrane. After the primary Ab reaction, these membranes were labeled by a method using donkey anti-rabbit IgG-HRP, anti-goat IgG-HRP Ab (Santa Cruz Biotechnology) and then detected by a Pierce ECL Western blotting substrate (Pierce, Rockford, IL, USA).

Statistical analysis. The significance of the differences between the control and experimental groups was determined using the Mann-Whitney U test or Student's t-test depending on the data distribution. Statistical analysis was performed using the Excel 
A

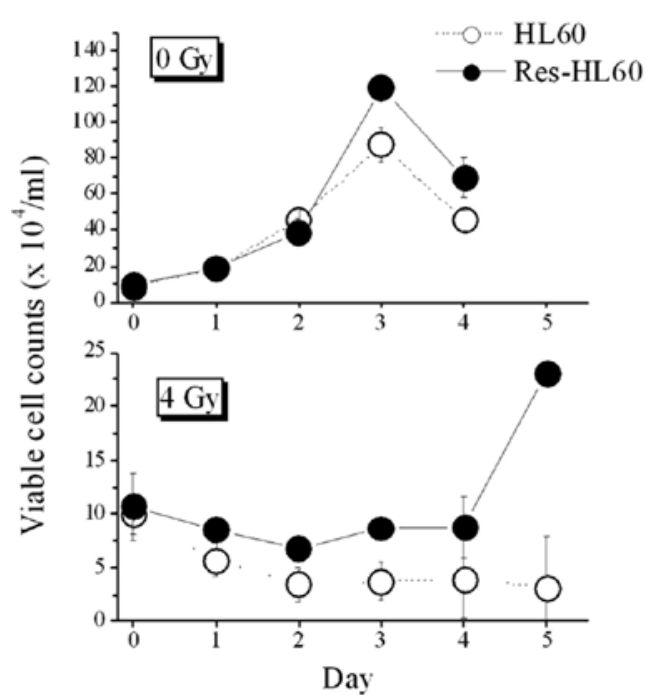

B

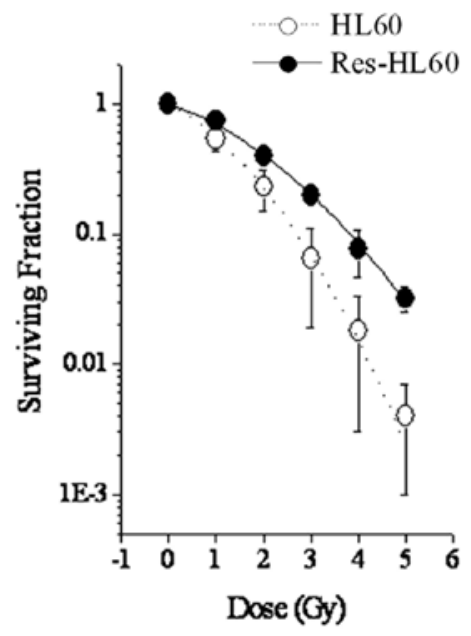

Figure 1. Survival and proliferation of HL60 and Res-HL60 cells. (A) The proliferation activity in HL60 and Res-HL60 cells. Number of cell counts were examined after having adjusted cell counts to $100,000 / \mathrm{mm}^{3}$. Res-HL60 cells were increased $>1,000,000 / \mathrm{mm}^{3}$ (upper graph). Number of cell counts after exposure to 4 Gy of $\mathrm{X}$-ray radiation (lower graph). Res-HL60 cells started proliferating 5 days after exposure to X-ray radiation. (B) Survival curves of the clonogenic assay for HL60 and Res-HL60 cells exposed to X-rays. Survival rates are higher in Res-HL60 cells than in HL60 cells on exposure to >2 Gy of radiation.

2003 (Microsoft $^{\circledR}$, Redmond, WA) with the add-in software Statcel 2.

\section{Results}

Differences in sensitivity to radiation and morphological changes between HL60 and Res-HL60 cells. In radiation therapy for leukemia, patients were subjected to radiation at $4 \mathrm{~Gy}$ x 3 days (12). In our experiment, however, because HL60 cells did not survive irradiation by this method, cells were irradiated at 4 Gy at intervals 1 week apart in order to establish a resistant strain (Res-HL60) by achieving a total dose of $16 \mathrm{~Gy}$. To investigate cell proliferation, the number of cell counts was determined after having adjusted the cell count to $1.0 \times 10^{5} / \mathrm{ml}$. The number of Res-HL60 cells increased more than $1.0 \times 10^{6} / \mathrm{ml}$, whereas the growth of HL60 cells was restrained (Fig. 1). Fig. 1A shows the cell counts after exposure to 4-Gy X-ray radiation. The number of HL60 cells decreased with time. However, Res-HL60 cells started proliferating at 5 days after exposure to X-ray radiation. As shown in Fig. 1B, Res-HL60 cells have remarkable resistance to radiation as compared with HL60 cells. The cell sizes were measured on the photographs, which were taken through a microscope. The size of Res-HL60 cells is smaller than that of HL60 cells $(\mathrm{p}<0.01)$ (Table I).

Cell cycle distribution in HL60 and Res-HL60 cells. Because the cell cycle is arrested when cells are exposed to genotoxic stress such as X-rays, the cell cycle distributions were investigated by using a Cell Lab Quanta Sc MPL (Fig. 2). X-ray irradiation induced G2/M arrest in both HL60 and Res-HL60 cells. However, among cells in the sub-G1 phase, the number of HL60 cells showing DNA fragmentation increased in a dosedependent manner on exposure to X-rays (Fig. 2). In contrast, marked blockages at the $\mathrm{G} 2 / \mathrm{M}$ phase and not at the sub-G1
Table I. Analysis of cell size.

\begin{tabular}{lcc}
\hline Cell type & Average $(\mu \mathrm{m})$ & SD \\
\hline HL60 cells & 17.26 & 1.95 \\
Res-HL60 cells & 12.86 & 1.10 \\
\hline
\end{tabular}

Statistically significant difference $(\mathrm{p}<0.01)$ was seen in cell size between HL60 cells and Res-HL60 cells. SD, standard deviation.

phase were induced in Res-HL60 cells (Fig. 2). These results show that differences in cell cycle patterns exist between HL-60 and Res-HL60 cells.

Caspase-3 activity in HL60 and Res-HL60 cells. Because apoptotic cells with degraded DNA are represented by the sub-G1 phase, we examined the expression of caspase-3, an executioner of the final phase of apoptosis. The expression of active caspase- 3 in HL60 cells was largely increased with irradiation. Although the expression of active caspase-3 in Res-HL60 cells was also upregulated by irradiation, the expression level was significantly lower than that observed in HL60 cells (Fig. 3). This result is in line with the finding of the sub-G1 population, and suggests that Res-H160 cells are resistant to X-ray irradiation and do not undergo apoptosis on exposure to X-rays.

Differences in $\gamma-H 2 A X$ transition and DNA fragmentation between HL60 and Res-HL60 cells. As phosphorylation of histone $\mathrm{H} 2 \mathrm{AX}$ at Ser $139(\gamma-\mathrm{H} 2 \mathrm{AX})$ represents one of the earlier steps in DSBs and is used as a sensitive measure of DSBs, transition of $\gamma-\mathrm{H} 2 \mathrm{AX}$ and DNA fragmentations were analyzed. As shown in Fig. 4, the level of $\gamma$-H2AX immediately increased in both cell lines after exposure to radiation, 


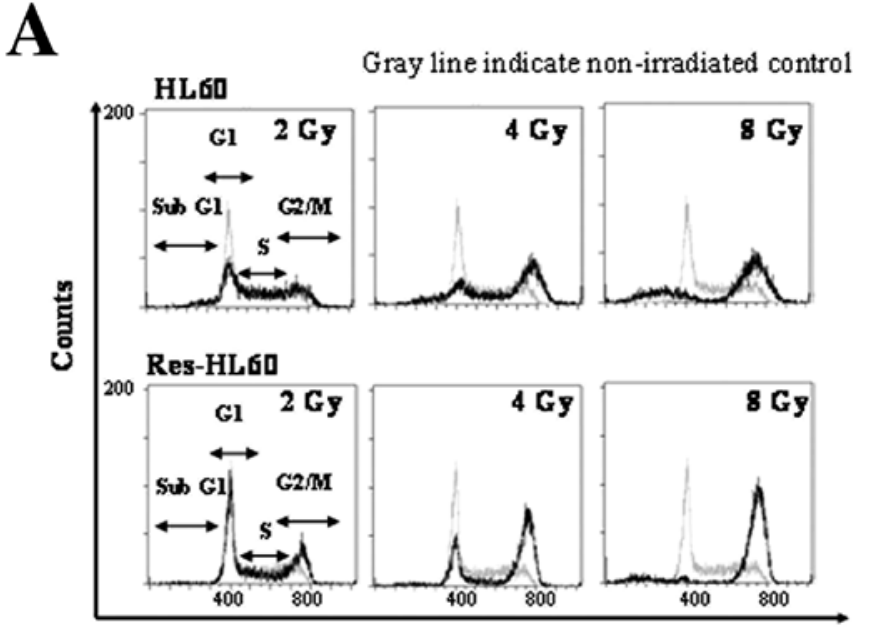

PI (DNA contents)

B

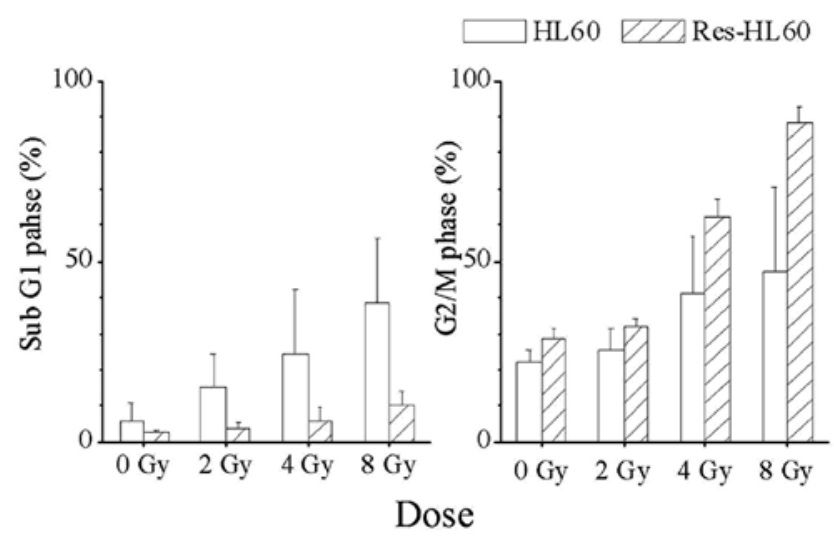

Figure 2. Flow cytometric patterns for cell cycle in HL60 and Res-HL60 cells exposed to X-rays. (A) Representative cytograms are shown. (B) Quantitative analysis of cell cycle distribution showing flow cytometric pattern. The percentage of cells in the sub-G1 phase increases with an increase in the X-ray dose. On the other hand, the percentage of Res-HL60 cells in the G2/M phase remarkably increases with an increase in the X-ray dose. although the levels were consistently lower in Res-HL60 cells than in HL60 cells. Furthermore, a single peak indicating $\gamma$-H2AX transition was observed in Res-HL60 cells, whereas the level of $\gamma$-H2AX increased again in HL60 cells at 120 min after irradiation. In terms of DNA fragments, those amounts were increased in HL60 cells and not in Res-HL60 cells at $2 \mathrm{~h}$ after irradiation (Fig. 4) (13).

Differences in transition of ATM and chkl between HL60 and Res-HL60 cells. Because DNA fragmentation occurred even at $2 \mathrm{~h}$ after irradiation (Fig. 4), we focused on the repair process at earlier time-points $(30,60,90$, and $120 \mathrm{~min}$ after irradiation). Western blot analysis showed that under non-irradiated conditions, the expression of ATM, which is associated with the DNA damage sensor and repair, and $\mathrm{ChK}$, which has key roles in checkpoint signaling, was higher in Res-HL60 cells than in HL60 cells (Fig. 5). Surprisingly, Chk1 showed contrasting activities in HL60 and Res-HL60 cells. Although Chk1 in HL60 cells was activated after irradiation, the expression of Chk1 in Res-HL60 cells decreased. Furthermore, there were few differences in Chk2 activities between the cell lines. Chk2 protein expression in both HL60 and Res-HL60 cells increased 30 min after X-ray radiation and started decreasing after $60 \mathrm{~min}$. On the other hand, DNA-PKcs (Ser 2056), which phosphorylates $\mathrm{H} 2 \mathrm{AX}$ during apoptotic DNA fragmentation, was detected in HL60 cells but not in Res-HL60 cells at $120 \mathrm{~min}$ after irradiation (Fig. 5). These results are appropriate and strongly support the transition of $\gamma$-H2AX in HL60 cells.

The effect of ATM inhibitor or DNA-PKcs inhibitor. To examine the relation between ATM and DNA-PKcs, caffeine (inhibitor of ATM activity) or NU7026 (DNA-PKcs specific inhibitor) was used. The clonogenic survival rate was investigated after using each of the inhibitors. With regard to surviving fraction (4 Gy), each inhibitor decreased the survival of Res-HL60 cells (Fig. 6). The results of flow cytometric patterns for cell cycle of Res-HL60 using these inhibiters are shown in Fig. 6B. When

\section{B}
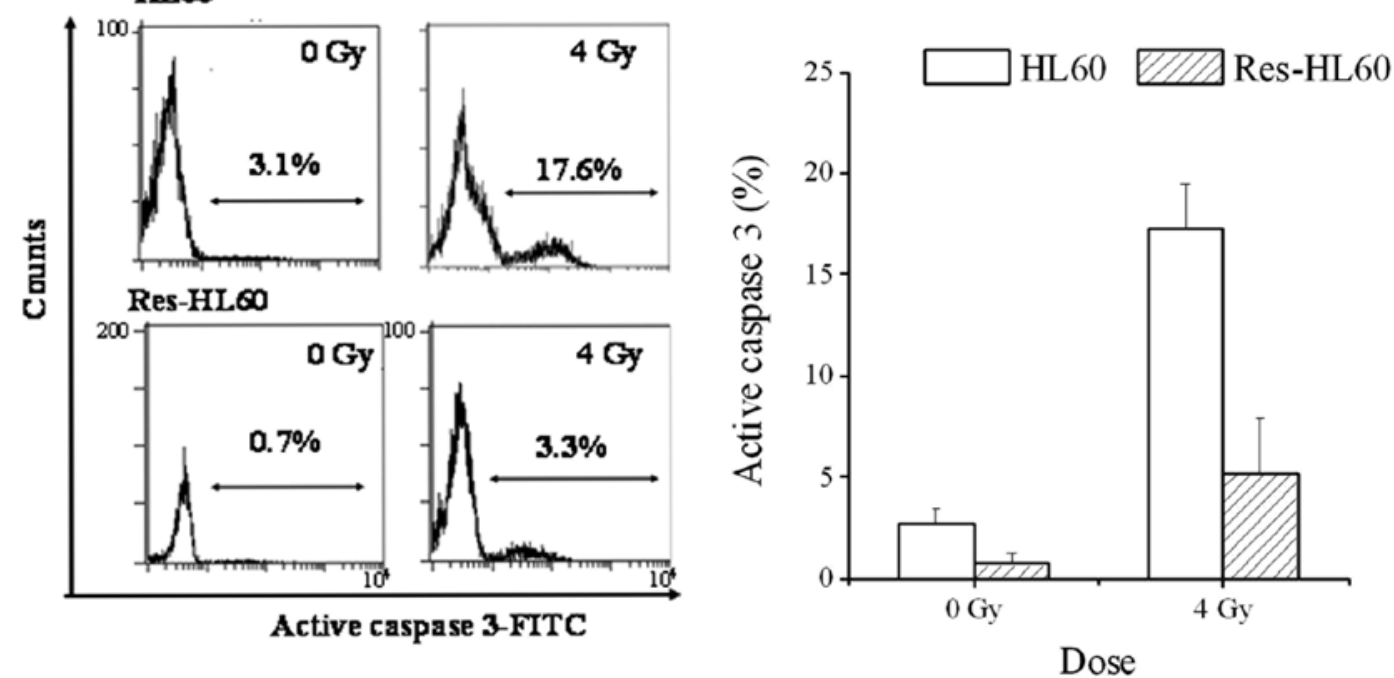

Figure 3. Flow cytometric analysis of caspase-3 activity in HL60 and Res-HL60 cells exposed to X-rays. (A) Sub-G1 is left and Res-HL60. (B) Graph of caspase-3 activity in flow cytometric analysis. Caspase-3 activity increases to a lesser extent in Res-HL60 cells than in HL60 cells. 
A

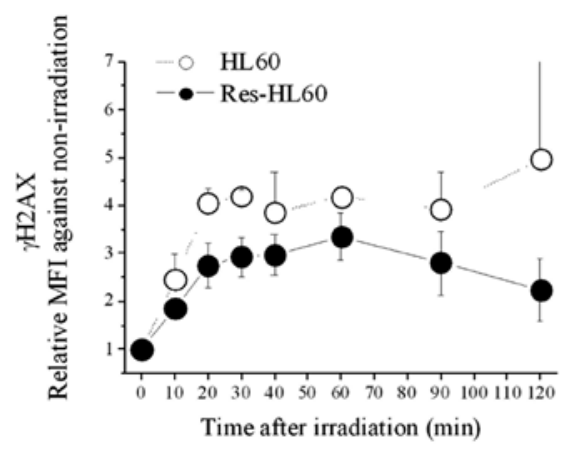

B

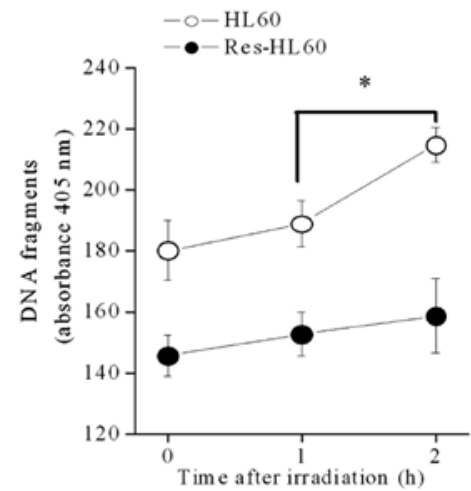

Figure 4. Analysis of DSBs and DNA fragmentation. (A) The result of $\gamma$-H2AX measurement by flow cytometry. Similar changes in HL60 and Res-HL60 cells are shown until $90 \mathrm{~min}$ of exposure to X-ray radiation. However, the relative mean fluorescence intensity (MFI) of $\gamma$-H2AX increases in HL60 cells after 120 min of exposure to X-ray radiation. (B) DNA fragmentation also increases at $120 \mathrm{~min}$ after exposure to X-ray radiation. These 2 values of Res-HL60 cells do not show a remarkable increase even at $120 \mathrm{~min}$ after exposure to $\mathrm{X}$-ray radiation.
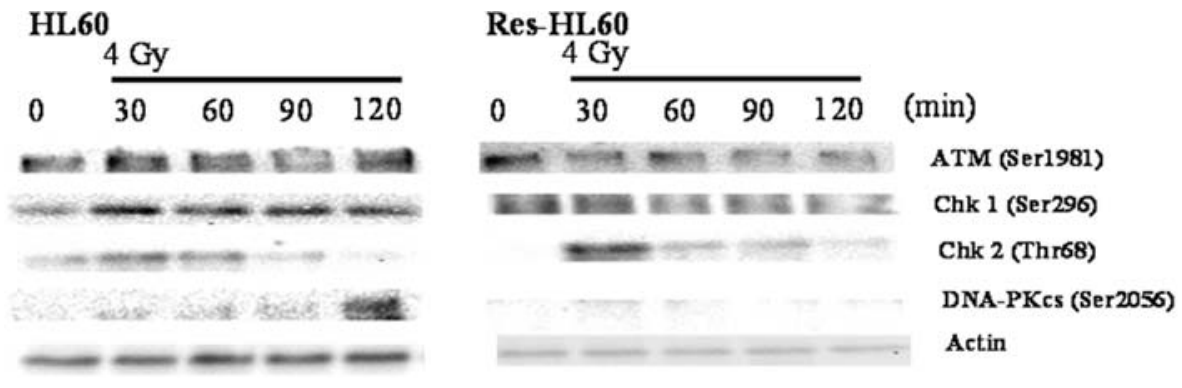

Figure 5. Western blot analysis of DSB repair-related proteins. Remarkable expression of DNA-PKcs (Ser 2056) was observed in HL60 cells. Chk1 activity in Res-HL60 cells decreases after exposure to X-ray radiation.

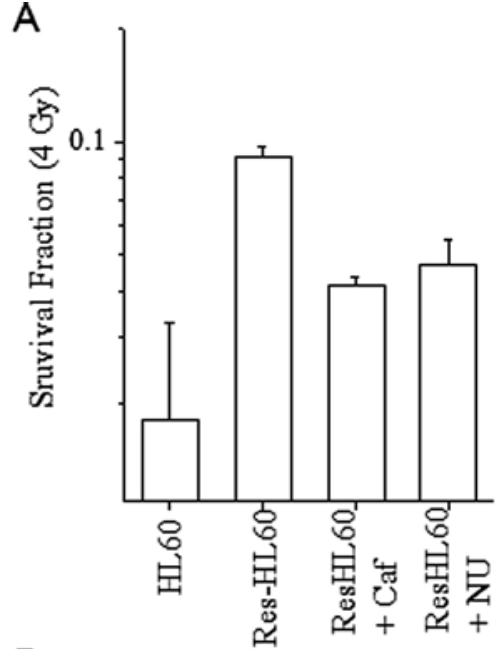

B

Res-HL60

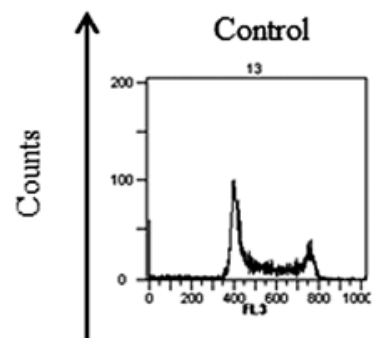

4 Gy

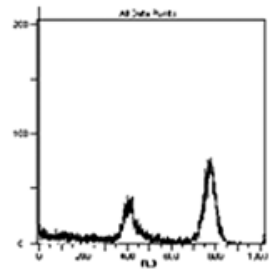

Figure 6. Analysis of clonogenic survival rate and cell cycle profile using caffeine (inhibitor of ATM activity) or NU7026 (DNA-PKcs specific inhibitor). Clonogenic survival rate in the presence of caffeine (inhibitor of ATM activity) or NU7026 (DNA-PKcs specific inhibitor). With regard to the surviving fraction (4 Gy), both inhibitors caused a reduction in the survival of Res-HL60 cells. (B) Flow cytometric patterns for cell cycle in Res-HL60 cells in the presence of caffeine (inhibitor of ATM activity) or NU7026 (DNA-PKcs specific inhibitor). The population of cells in the G2/M phase decreased after exposure to radiation in the presence of caffeine. There is no remarkable change in flow cytometric patterns of cells exposed to X-ray radiation alone and those exposed to X-ray radiation in the presence of $\mathrm{NU} 7026$. 
cells were exposed to radiation in the presence of caffeine, the G2/M checkpoint was effectively abrogated and the sub-G1 phase was prolonged as compared with in the case when cells were exposed to radiation alone in the absence of caffeine. On the other hand, NU7026 had no impact on the G2/M checkpoint. A similar pattern was observed in the presence and absence of NU7026.

\section{Discussion}

The present study focused on the acquisition of resistance to radiation, which is often observed in radiation therapy. There are very few studies investigating DNA repair and DSBs in HL60 cells (14). Res-HL60 cells established in our study were different from the cells from which they originated in terms of size, and DNA repair and proliferation activities. Furthermore, Res-HL60 cells not only proliferated on the fifth day after exposure to radiation but also showed a high survival rate in the clonogenic assay (Fig. 1). It is considered that tumors recur in this clinical state.

Exposure to X-rays induces DNA damage, and unrepaired DNA damage leads to apoptosis. The findings of the sub-G1 population and active caspase- 3 expression demonstrated that exposure to X-rays induced apoptosis in HL60 cells (Figs. 2 and 3). Furthermore, apoptosis induced by irradiation in HL60 cells warrants DNA-PKcs (Ser 2056) phosphorylation (Fig. 5). Several autophosphorylation sites have been mapped in the DNA-PKcs protein, and the most important clusters are found between residues 2609 and 2647 (ABCDE cluster) and between residues 2023 and 2056 (PQR cluster). The phosphorylation of the ABCDE cluster is found to specifically stimulate the processing and joining of DNA ends, whereas $\mathrm{PQR}$ phosphorylation reduced the level of DNA end processing (15). On the other hand, these apoptotic findings were not observed in Res-HL60 cells; this strongly demonstrates that Res-HL60 cells acquire resistance to radiation-induced apoptosis. It is noteworthy that the phosphorylation of DNA-PKcs occurred only at $120 \mathrm{~min}$ after irradiation. Furthermore, the increase in the level of $\gamma-\mathrm{H} 2 \mathrm{AX}$ in HL60 cells at 120 min may indicate the progression of apoptosis because $\gamma-\mathrm{H} 2 \mathrm{AX}$ occurs in cases of DNA fragmentation due to apoptosis (16). Therefore, these data may indicate that the fate of irradiated-cells could be determined within 120 min of irradiation.

Because HL60 cells are p53-mutated cells, exposure to radiation would lead to cell cycle arrest in the G2/M phase. Given that apoptosis is less frequent in Res-HL60 cells, as mentioned above, it is assumed that the repair is carried out in this $\mathrm{G} 2 / \mathrm{M}$ phase. Cells with defective p53 lack arrest in the G1 phase but show a block in the G2/M phase. Consistent with DNA repair, increased resistance to radiation with a prolonged arrest in the G2/M phase was shown (17). Regulation of checkpoint signaling by Chk1 and Chk2 is important for the repair process to arrest the cell cycle, and induce DNA repair and maintain DNA stability. Although Chk2 is regulated by ATM, Chk1 is activated in an ATM-dependent and Rad3-related (ATR)-dependent manner (18). ATM primarily initiates HR repair responses to DSBs and is recruited at damage sites by the Mre11-Rad50-Nbs1 complex. In contrast, ATR forms a complex with ATR-interacting protein (ATRIP) and is recruited to regions of replication protein A (RPA)-coated single-strand DNA (ssDNA) (19). Because HR repair process first needs to produce ssDNA at the end of damaged sites in order to interact with the sister chromatin, ATR is recruited not only to single-strand breaks and replication stresses but also to ssDNA as observed in the DSBs repair process (20). Chk1 is probably the key regulator in the survival and acquisition of resistance to radiation in Res-HL60 cells. Because there were few differences in the phosphorylated forms of Chk2 under various conditions, except for the progression of Chk1 phosphorylation in Res-HL60 cells, Chk1 could regulate the fate of irradiated cells (Fig. 6). Morgan et al reported that G(2) checkpoint abrogation and HR repair inhibition both contribute to radiosensitization by Chk1 inhibition (21). It is known that Chk1 regulates the S and G2/M checkpoints, and accordingly, the results are consistent with the fact that $\mathrm{G} 2$ arrest was pronounced in the resistant strain in our study (22).

It is unclear why the higher expression levels of ATM in Res-HL60 cells under normal conditions are associated with the radio-resistance of Res-HL60. However, treatment of cells with caffeine (an inhibitor of ATM activity) resulted in the abolition of G2/M arrest and promotion of the sub-G1 phase; this indicates that ATM is indispensable for $\mathrm{G} 2 / \mathrm{M}$ arrest and the inhibition of apoptosis by irradiation. On the other hand, treatment of cells with NU7026 (a DNA-PKcs specific inhibitor) retained the G2/M arrest under irradiation. To consider that both inhibitors decreased the survival of Res-HL60 cells, the DNA repair ability of Res-HL60 cells may occur in both the presence and absence of G2/M arrest. Furthermore, our observation that the level of $\gamma$-H2AX was reduced within $120 \mathrm{~min}$ in Res-HL60 cells also indicates that DNA damage is repaired in the early stage.

After radiation therapy, some patients may develop a local tumor relapse, which may be related to the sub-clinical focuses and resistant cell population, indicating poor prognosis (23). Because the radiation dose may be lethal in the case of cancer relapse after radiotherapy, chemotherapy rather than a second dose of radiation therapy would be preferred in such cases. Therefore, it is necessary to explore the problem of re-proliferation of radioresistant cells. In the present study, the radioresistant strains established by us showed resistance to radiation-induced apoptosis, probably because of their high DNA repair ability. Therefore, if the expression of radioresistance starts with the enhancement of a repair system immediately after irradiation and decreases the effect of radiation treatment in a radioresistant strain, treatment such as the addition of caffeine that inhibits this repair stage is considered to be effective.

\section{Acknowledgements}

This study was supported by a grant to Hirosaki University Institutional Research (2010).

\section{References}

1. Maity A, Kao GD, Muschel RJ and McKenna WG: Potential molecular targets for manipulating the radiation response. Int $\mathbf{J}$ Radiat Oncol Biol Phys 37: 639-653, 1997.

2. Lieber MR: The mechanism of double-strand DNA break repair by the nonhomologous DNA end-joining pathway. Annu Rev Biochem 79: 181-211, 2010.

3. Mahaney BL, Meek K and Lees-Miller SP: Repair of ionizing radiation-induced DNA double-strand breaks by non-homologous end-joining. Biochem J 417: 639-650, 2009. 
4. Ogiwara H, Ohuchi T, Ui A, Tada S, Enomoto T and Seki M: Ctf18 is required for homologous recombination-mediated double-strand break repair. Nucleic Acids Res 35: 4989-5000, 2007.

5. Shrivastav M, De Haro LP and Nickoloff JA: Regulation of DNA double-strand break repair pathway choice. Cell Res 18: 134-147, 2008.

6. Dobbs TA, Tainer JA and Lees-Miller SP: A structural mode for regulation of NHEJ by DNA-PKcs autophosphorylation.DNA Repair (Amst) 9: 1307-1314, 2010.

7. Foray N, Arlett CF and Malaise EP: Radiation-induced DNA double-strand breaks and the radiosensitivity of human cells: a closer look. Biochimie 79: 567-575, 1997.

8. Chavaudra N, Bourhis J and Foray N: Quantified relationship between cellular radiosensitivity, DNA repair defects and chromatin relaxation: a study of 19 human tumour cell lines from different origin. Radiother Oncol 73: 373-382, 2004.

9. Nishioka T, Yasuda M, Takeshima T, et al: Radiation-induced cancer cell repopulation: a possible mechanism implied by experiments using transplantable mouse-derived sarcoma cell line. Cell Struct Funct 36: 13-20, 2011.

10. Kashiwakura I, Kuwabara M, Inanami O, et al: Radiation sensitivity of megakaryocyte colony-forming cells in human placental and umbilical cord blood. Radiat Res 153: 144-152, 2000.

11. Takahashi Y, Hazawa M, Takahashi K, Nishizawa A, Yoshizawa A and Kashiwakura I: Suppressive effects of liquid crystal compounds on the growth of the A549 human lung cancer cell line. Invest New Drugs 29: 659-665, 2011.

12. Carruthers SA and Wallington MM: Total body irradiation and pneumonitis risk: a review of outcomes. Br J Cancer 90: 20802084, 2004.

13. Shinozaki K, Hosokawa Y, Hazawa M, et al: Ascorbic acid enhances radiation-induced apoptosis in an HL60 human leukemia cell line. J Radiat Res 52: 229-237, 2011.
14. Terry SY, Riches AC and Bryant PE: A role for topoisomerase II alpha in the formation of radiation-induced chromatid breaks. $\mathrm{Br}$ J Cancer 99: 670-674, 2008

15. Weterings E, Verkaik NS, Keijzers G, et al: The Ku80 carboxy terminus stimulates joining and artemis-mediated processing of DNA ends. Mol Cell Biol 29: 1134-1142, 2009.

16. Kurose A, Tanaka T, Huang X, Traganos F, Dai W and Darzynkiewicz Z: Effects of hydroxyurea and aphidicolin on phosphorylation of ataxia telangiectasia mutated on Ser 1981 and histone H2AX on Ser 139 in relation to cell cycle phase and induction of apoptosis. Cytometry A 69: 212-221, 2006.

17. Bache M, Pigorsch S, Dunst J, et al: Loss of G2/M arrest correlates with radiosensitization in two human sarcoma cell lines with mutant p53. Int J Cancer 96: 110-117, 2001.

18. Smith J, Tho LM, Xu N and Gillespie DA: The ATM-Chk2 and ATR-Chk1 pathways in DNA damage signaling and cancer. Adv Cancer Res 108: 73-112, 2010.

19. Reinhardt HC and Yaffe MB: Kinases that control the cell cycle in response to DNA damage: Chk1, Chk2, and MK2. Curr Opin Cell Biol 21: 245-255, 2009.

20. Dotiwala F, Harrison JC, Jain S, Sugawara N and Haber JE: Mad2 prolongs DNA damage checkpoint arrest caused by a double-strand break via a centromere-dependent mechanism. Curr Biol 20: $328-332,2010$.

21. Morgan MA, Parsels LA, Zhao L, et al: Mechanism of radiosensitization by the Chk1/2 inhibitor AZD7762 involves abrogation of the G2 checkpoint and inhibition of homologous recombinational DNA repair. Cancer Res 70: 4972-4981, 2010.

22. Peng G and Lin SY: Exploiting the homologous recombination DNA repair network for targeted cancer therapy. World J Clin Oncol 2: 73-79, 2011.

23. Shi D, Shi G, Huang G, Zhang J and Lartigau E: Chemosensitivity of radioresistant cells in the multicellular spheroids of A549 lung adenocarcinoma. J Exp Clin Cancer Res 28: 72, 2009. 